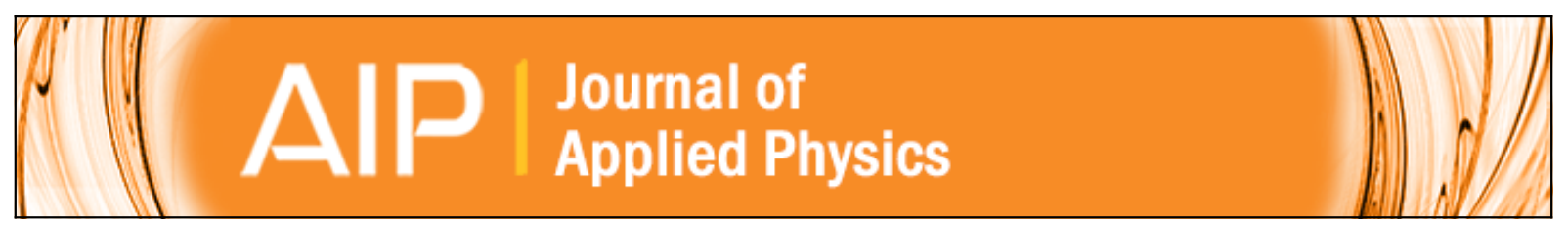

\title{
Electronegativity and doping in semiconductors
}

U. Schwingenschlögl, A. Chroneos, C. Schuster, and R. W. Grimes

Citation: Journal of Applied Physics 112, 046101 (2012); doi: 10.1063/1.4747932

View online: http://dx.doi.org/10.1063/1.4747932

View Table of Contents: http://scitation.aip.org/content/aip/journal/jap/112/4?ver=pdfcov

Published by the AIP Publishing

$\stackrel{A}{A} \mathbb{P} P$ Re-register for Table of Content Alerts

Create a profile.

Sign up today! 


\title{
Electronegativity and doping in semiconductors
}

\author{
U. Schwingenschlögl, ${ }^{1}$ A. Chroneos, ${ }^{2, a)}$ C. Schuster, ${ }^{3}$ and R. W. Grimes ${ }^{2}$ \\ ${ }^{1}$ PSE Division, KAUST, Thuwal 23955-6900, Saudi Arabia \\ ${ }^{2}$ Department of Materials, Imperial College London, London SW7 2BP, United Kingdom \\ ${ }^{3}$ Institut für Physik, Universität Augsburg, 86135 Augsburg, Germany
}

(Received 27 March 2012; accepted 20 July 2012; published online 23 August 2012)

\begin{abstract}
Charge transfer predicted by standard models is at odds with Pauling's electronegativities but can be reconciled by the introduction of a cluster formation model [Schwingenschlögl et al., Appl. Phys. Lett. 96, 242107 (2010)]. Using electronic structure calculations, we investigate $p$ - and $n$-type doping in silicon and diamond in order to facilitate comparison as $\mathrm{C}$ has a higher electronegativity compared to Si. All doping conditions considered can be explained in the framework of the cluster formation model. The implications for codoping strategies and dopant-defect interactions are discussed. (C) 2012 American Institute of Physics. [http://dx.doi.org/10.1063/1.4747932]
\end{abstract}

To satisfy the requirement of advanced performance, the microelectronics industry is focused on the introduction of materials to substitute silicon. An aspect that can remain constant in the future nanoelectronic devices is doping to form both $p$-type (acceptor) and $n$-type (donor) doped regions. The reduction of the dimensions of devices to a few nanometers requires the absolute control of the properties of dopants in semiconductors, including their surfaces and interfaces. In the past few years, numerous materials and structures have been considered, such as new forms of carbon (fullerenes, nanotubes, graphene), germanium, and diamond. ${ }^{1-6}$

Doping in group-IV semiconductor materials such as $\mathrm{Si}$ can be achieved by the incorporation of trivalent atoms such as gallium ( $p$-type doping) or with the introduction of pentavalent atoms such as phosphorous ( $n$-type doping). The picture commonly described is that in the case of $n$-type doping an electron is donated by the dopant atom (donor) to the lattice, whereas the opposite is the case for $p$-type doping. Nevertheless, if one considers the Pauling electronegativities $^{7,8}$ of the dopants as compared to the host atoms the picture is not consistent to what would be expected. That is, an electron would transfer from the $\mathrm{Si}$ atoms (electronegativity 1.90) to the phosphorous atom (electronegativity 2.19) and from the Ga atom (electronegativity 1.81) to the $\mathrm{Si}$ atoms. The recently introduced cluster formation model ${ }^{9,10}$ bridges the gap between the standard picture in semiconductors and electronegativities by considering that it is the cluster between the dopant atom and the nearest neighbor host atoms that donates (or accepts) the electron and not the isolated dopant. For example, if one considers an $n$-type dopant, such as $\mathrm{P}$ in $\mathrm{Si}$, it does not donate an electron to the conduction band as commonly expected nor does it accept an charge as the electronegativities would dictate ( $\mathrm{P}$ is more electronegative than $\mathrm{Si}$ ). The $\mathrm{P}$ dopant forms a cluster with its four nearest neighbor $\mathrm{Si}$ atoms $\left(\mathrm{PSi}_{4}\right)$ and through the redistribution of charge in this cluster the electron is donated to the system.

Here we will consider the cluster formation model to gain insight into the $p$ - and $n$-type doping of silicon and

\footnotetext{
a) Author to whom correspondence should be addressed. Electronic address: alexander.chroneos@imperial.ac.uk.
}

diamond. Silicon is the archetypal semiconductor, whereas diamond can be an ideal material for many electronic applications as it has a wide band gap, high carrier mobility, thermal conductivity, and low dielectric constant. ${ }^{11-13}$ The aim is to compare these two isostructural materials since they have significantly different electronegativities.

Established electronic structure calculations (WIEN2K) ${ }^{14}$ based on density functional theory are used to investigate the effects of doping in silicon and diamond. These methods have adequately been applied to study charge transfer effects. ${ }^{15}$ Here we employ a $2 \times 2 \times 2$ supercell of the cubic diamond unit cell ( $\mathrm{Si}$ or $\mathrm{C}$ ). This supercell of 64 atoms is doped by inserting one impurity atom (B, Ga, N, or As) at a substitutional site. Therefore, we consider a high doping level of $1.6 \%$. In the calculations of the $\mathrm{C}$ and $\mathrm{Si}$ systems, we use plane wave cutoffs given by $\mathrm{RK}_{\max }=8$ and $\mathrm{RK}_{\max }=7$, respectively, as well as muffintin radii of $2.21 \AA$ for $\mathrm{Si}$ and $1.41 \AA$ for $\mathrm{C}$. The $\mathrm{B} 2 \mathrm{~s}, \mathrm{~N} 2 \mathrm{~s}$, C 2 s, P $3 \mathrm{~s}$, As $3 \mathrm{~d} / 4 \mathrm{~s}$, and Ga $3 d$ atomic orbitals are assumed to be semicore states, while the B $2 p / 3 s, C 2 p / 3 s, N 2 p / 3 s$, Si 3s/3p, P 3p/4s, As $4 p / 4 d / 5 s$, and Ga $4 s / 4 p / 4 d$ orbitals are treated as valence states. We refined the k-mesh for the Brillouin zone integration until the results showed convergence. For the $\mathrm{C}$ and $\mathrm{Si}$ systems, this is the case for 39 and $24 \mathrm{k}$-points within the irreducible wedge of the Brillouin zone, respectively. Finally, the exchange correlation functional is treated in the generalized gradient approximation.

For the $p$-type dopants, we introduced gallium (Figs. 1(a) and 1(b)) and boron (Figs. 1(c) and 1(d)) for silicon and diamond, respectively. Both boron and gallium have lower electronegativities than the respective host lattices (electronegativities: C 2.55, B 2.04, Si 1.90, and Ga 1.81). Considering boron-doped diamond, an acceptor region can be achieved with an acceptor level about $0.37 \mathrm{eV}$ below the valence band maximum. ${ }^{16}$ From electronegativity considerations alone in diamond, it is expected that charge will be transferred from the less electronegative boron to carbon. Changes in electron density (a difference map) have been utilized here to consider the charge redistribution (in the figures red indicates where charge is lost) associated with the introduction of a dopant in the lattice. This is what is calculated if we consider the difference between a neutral 


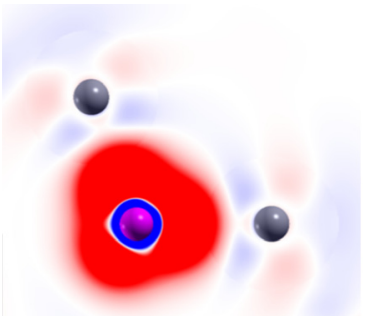

(a)

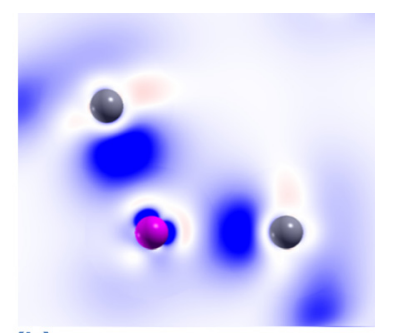

(b)

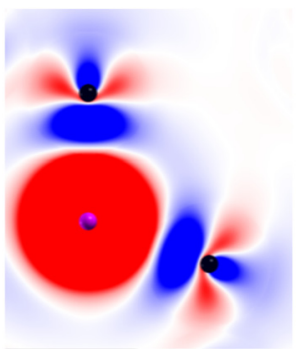

(c)

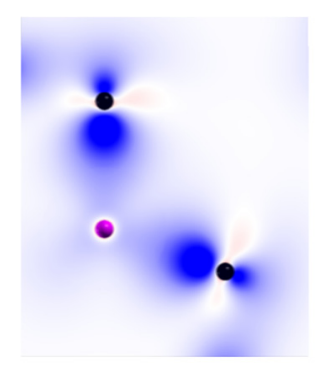

(d)

FIG. 1. Electron density map of the (110) plane of diamond showing the difference between (a) a neutral $\mathrm{Si}_{64}$ supercell and a neutral $\mathrm{GaSi}_{63}$ supercell, (b) a neutral $\mathrm{Si}_{64}$ supercell and a negatively charged $\mathrm{GaSi}_{63}{ }^{-}$supercell, (c) a neutral $\mathrm{C}_{64}$ supercell and a neutral $\mathrm{BC}_{63}$ supercell, and (d) a neutral $\mathrm{C}_{64}$ supercell and a negatively charged $\mathrm{BC}_{63}{ }^{-}$supercell. Large grey spheres mark the Ga atoms, small black spheres the B atoms, and large/small purple spheres the $\mathrm{Si} / \mathrm{C}$ atoms. Red color indicates where charge is lost.

$\mathrm{C}_{64}$ supercell and a neutral $\mathrm{BC}_{63}$ supercell (map of part of the (110) plane of diamond in Fig. 1(c)). Analogous results are obtained when considering gallium doped silicon (Fig. 1(a)). Figure 1(d) is the electron density difference map between a neutral $\mathrm{C}_{64}$ supercell and a negatively charged $\mathrm{BC}_{63}{ }^{-}$supercell. The latter cell has assumed an additional single charge that is not localized only at the $\mathrm{B}$ atom but in essence trapped by the $\mathrm{B}$ atom and its four nearest neighbor $\mathrm{C}$ atoms (i.e., the $\mathrm{BC}_{4}$ cluster). Similarly, in gallium-doped silicon, the charge is trapped by the $\mathrm{GaSi}_{4}$ cluster (Fig. 1(b)). In essence, what we demonstrate here is that the acceptor atom only establishes a suitable local environment that results in the attraction of the electronic charge via a more extended cluster. Furthermore, what is important for this behavior is the relative electronegativities of the dopant atoms with respect to the host atoms (and not the specific dopant or host atoms).

For the $n$-type dopants, we consider arsenic (Figs. 2(a) and 2(b)) and nitrogen (Figs. 2(c) and 2(d)) for silicon and diamond, respectively. Both dopants have higher electronegativities than the respective host lattices (electronegativities: C 2.55, N 3.05, Si 1.90, and As 2.18). Arsenic alongside phosphorous are common $n$-type dopants in silicon or related materials such as $\mathrm{Si}_{1-\mathrm{x}} \mathrm{Ge}_{\mathrm{x}}$ and germanium. ${ }^{17-19}$ Conversely, in diamond, there is no shallow donor, with both nitrogen and phosphorous being inappropriate. ${ }^{20-22}$ In more detail, nitrogen does not contribute to the conduction band but in turn leads to the formation of a lone electron pair on the nitrogen atom and a dangling bond with one of its nearest neighbor carbon atoms. The unpaired electron is localized more on the carbon nearest neighbor atom rather than on the nitrogen dopant. ${ }^{23}$ Essentially, nitrogen has a deep donor level (activation energy

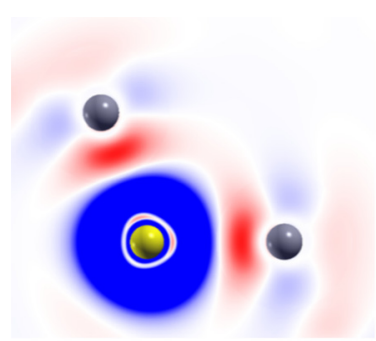

(a)

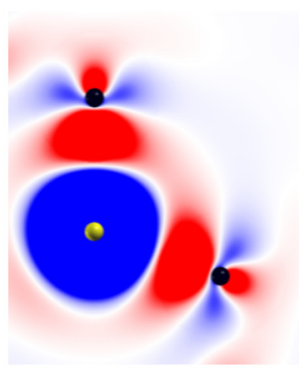

(c)

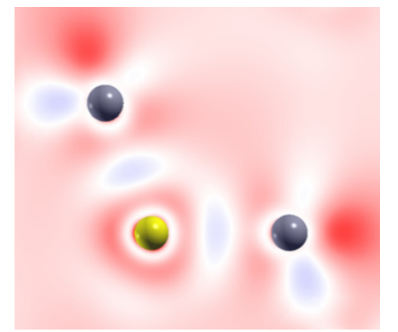

(b)

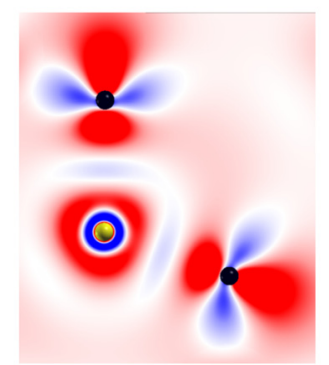

(d)
FIG. 2. Electron density map of the (110) plane of diamond showing the difference between (a) a neutral $\mathrm{Si}_{64}$ supercell and a neutral $\mathrm{AsSi}_{63}$ supercell, (b) a neutral $\mathrm{Si}_{64}$ supercell and a positively charged $\mathrm{AsSi}_{63}{ }^{+}$supercell, (c) a neutral $\mathrm{C}_{64}$ supercell and a neutral $\mathrm{NC}_{63}$ supercell, and (d) a neutral $\mathrm{C}_{64}$ supercell and a positively charged $\mathrm{NC}_{63}{ }^{+}$supercell. Large grey spheres mark the As atoms. Small black spheres mark the $\mathrm{N}$ atoms and large/small yellow spheres the $\mathrm{Si} / \mathrm{C}$ atoms. Red color indicates where charge is lost. For $\mathrm{N}$ doped diamond, a strong loss affects the $\mathrm{C}$ atoms, whereas $\mathrm{N}$ rather accumulates charge. The opposite picture is observed for As doped diamond.

of $1.7 \mathrm{eV}$ ), which in turn leads to negligible excitation of electrons into the conduction band at room temperature. ${ }^{20,22}$

Figure 2 shows the change in electron density between the $\mathrm{Si}_{64}$ (or $\mathrm{C}_{64}$ ) cell and the $\mathrm{AsSi}_{63}$ (or $\mathrm{NC}_{63}$ ) cell and therefore the charge redistribution associated with the introduction of the substitutional dopant. In Fig. 2, it is demonstrated that charge is lost (red color) from the neighboring host atoms (C or As) and is concentrated in the n-type dopants ( $\mathrm{N}$ or As). Notably, these host atom/dopant combinations have similar electronegativity ratios $(\mathrm{C} / \mathrm{N}=84 \%$ and $\mathrm{Si} / \mathrm{As}=87 \%)$ and this is reflected in their qualitatively similar electron density maps (refer to Fig. 2). The higher electronegativity difference of nitrogen with carbon (compared to arsenic with silicon) leads to the accumulation of more charge around nitrogen and a stronger loss around carbon atoms. This is illustrated in Figs. 2(b) and 2(d), which show (i) the difference between a neutral $\mathrm{Si}_{64}$ supercell and a positively charged $\mathrm{AsSi}_{63}{ }^{+}$supercell and (ii) the difference between a neutral $\mathrm{C}_{64}$ supercell and a positively charged $\mathrm{NC}_{63}{ }^{+}$supercell. For both host materials (Figs. 2(b) and 2(d)), it is evident that the missing electron is coming from the near neighbor host atoms (i.e., as expected by considering Pauling's electronegativities) rather than the pentavalent dopant (i.e., as expected by the standard model for $n$-type doping). Previous work ${ }^{9}$ has considered phosphorous doped silicon with the results being consistent (note: phosphorous has very similar electronegativity to arsenic). It is appropriate to propose that $n$-type doping in semiconductor materials irrespective of the host is via the promotion of an electron from a cluster consisting of the nearest 
neighbor host atoms and the dopant atom. In the materials considered here, these are the $\mathrm{AsSi}_{4}$ and $\mathrm{NC}_{4}$ clusters for silicon and diamond, respectively. The high electronegativity of nitrogen as compared to carbon can explain its poor donor properties in diamond ${ }^{16}$ as discussed in previous work. ${ }^{10}$

Our findings have important implications for dopantdefect interactions. To surpass difficulties in the doping of semiconductors defect engineering strategies such as codoping are commonly used. ${ }^{24,25}$ A recent example is the need to restrain donor atom diffusion (via a vacancy mechanism) in germanium by the use of carbon or fluorine codoping. The introduction of such codopants may lead to charge trapping as they do have larger electronegativities as compared to the host Ge atoms (electronegativity of Ge 2.01, C 2.55, F 3.98) or the $n$-type dopants (P, As, Sb considered). In that sense, the clusters consisting of donor-codopant atoms in germanium must be investigated in detail. In silicon, isovalent codoping has been recently used to defect engineer the formation and stability of the oxygen-vacancy pair (or A-center). ${ }^{26}$ These issues are presently under investigation from the viewpoint of the cluster formation model as the isovalent codopants of interest have significantly different electronegativities (hafnium 1.3 to lead 2.33) offering possibilities for tuning the properties. Another way forward is to consider alloy group-IV semiconductors such as the $\mathrm{Si}_{1-\mathrm{x}-\mathrm{y}} \mathrm{Ge}_{\mathrm{x}} \mathrm{Sn}_{\mathrm{y}}$ alloys (i.e., change the electronegativity of the host atoms around the dopant). ${ }^{27,28}$

Considering diamond, popular codoping strategies propose the formation of donor-acceptor clusters. ${ }^{29-31}$ Donoracceptor clusters are actively considered as they increase the solubility of dopants (via the Coulomb attraction between the donor and acceptor atoms) and decrease the defect level concentration (via donor and acceptor level repulsion). Similar donor-acceptor clusters (the N-B-N cluster) have been proposed previously. ${ }^{29}$ The Pauling electronegativity of $\mathrm{B}$ is significantly lower than that of nitrogen. In fact, only fluorine, oxygen, and chlorine have higher Pauling electronegativities. From these considerations, codoping strategies in diamond would benefit more if the donor atom was phosphorous for which there are numerous candidate codopants with higher electronegativities. ${ }^{10}$

We have considered the doping of two isostructural host materials, silicon and diamond, which have significantly different electronegativities. For both materials, the results are qualitatively similar and depend on the differences of electronegativities with the dopants. For all the doping scenarios considered, the charge is accepted or donated not by the isolated dopants but by the cluster consisting of the dopant atom and the nearest neighbor host atoms. The electronegativity of codopants is therefore an important criterion when considering codoping strategies. In essence, one must form an appropriate nearest neighbor environment for the dopant.
We acknowledge financial support by the Deutsche Forschungsgemeinschaft (TRR 80).

${ }^{1}$ H. W. Kroto, J. R. Heath. S. C. O’Brien, R. F. Curl, and R. E. Smalley, Nature 318, 162 (1985).

${ }^{2}$ K. S. Novoselov, A. K. Geim, S. V. Morozov, D. Jiang, Y. Zhang, S. V. Dubonos, I. V. Grigorieva, and A. A. Firsov, Science 306, 666 (2004).

${ }^{3}$ S. Decoster, B. De Vries, U. Wahl, J. G. Correia, and A. Vantomme, J. Appl. Phys. 105, 083522 (2009).

${ }^{4}$ N. A. Stolwijk and L. Lerner, J. Appl. Phys. 110, 033526 (2011).

${ }^{5}$ H. Tahini, A. Chroneos, R. W. Grimes, and U. Schwingenschlögl, Appl. Phys. Lett. 99, 162103 (2011).

${ }^{6}$ A. Chroneos, J. Appl. Phys. 107, 076102 (2010).

${ }^{7}$ N. W. Ashcroft and N. D. Mermin, in Solid State Physics (Harcourt, 1976), Chap. 28, p. 577.

${ }^{8}$ L. Pauling, J. Am. Chem. Soc. 54, 3570 (1932).

${ }^{9}$ U. Schwingenschlögl, A. Chroneos, C. Schuster, and R. W. Grimes, Appl. Phys. Lett. 96, 242107 (2010).

${ }^{10}$ U. Schwingenschlögl, A. Chroneos, C. Schuster, and R. W. Grimes, J. Appl. Phys. 110, 056107 (2011).

${ }^{11}$ G. A. J. Amaratunga, Science 297, 1657 (2002).

${ }^{12}$ S. J. Sque, R. Jones, J. P. Goss, and P. R. Briddon, Phys. Rev. Lett. 92, 017402 (2004).

${ }^{13}$ J. O. Orwa, C. Santori, K. M. C. Fu, B. Gibson, D. Simpson, I. Aharanovich, A. Stacey, A. Cimmino, P. Balog, M. Markham, D. Twitchen, A. D. Greentree, R. G. Beausoleil, and S. Prawer, J. Appl. Phys. 109, 083530 (2011).

${ }^{14}$ P. Blaha, K. Schwarz, G. Madsen, D. Kvasicka, and J. Luitz, Wien2k: An Augmented Plane Wave Plus Local Orbitals Program for Calculating Crystal Properties (TU Vienna, Vienna, 2001).

${ }^{15}$ U. Schwingenschlögl and C. Schuster, Phys. Rev. Lett. 102, 227002 (2009).

${ }^{16}$ R. M. Chrenko, Phys. Rev. B. 7, 4560 (1973).

${ }^{17}$ A. Chroneos, C. Jiang, R. W. Grimes, U. Schwingenschlögl, and H. Bracht, Appl. Phys. Lett. 95, 112101 (2009).

${ }^{18}$ A. Chroneos, H. Bracht, R. W. Grimes, and B. P. Uberuaga, Appl. Phys. Lett. 92, 172103 (2008).

${ }^{19}$ G. Impellizzeri, S. Boninelli, F. Priolo, E. Napolitani, C. Spinella, A. Chroneos, and H. Bracht, J. Appl. Phys. 109, 113527 (2011).

${ }^{20}$ S. Koizumi, M. Kamo, Y. Sato, H. Ozaki, and T. Inuzaka, Appl. Phys. Lett. 71, 1065 (1997).

${ }^{21}$ B. B. Li, M. C. Tosin, A. C. Peterlevitz, and V. Baranauskas, Appl. Phys. Lett. 73, 812 (1998).

${ }^{22}$ H. Okazaki, R. Yoshida, T. Muro, T. Nakamura, T. Wakita, Y. Muraoka, M. Hirai, H. Kato, S. Yamasaki, Y. Takano, S. Ishii, T. Oguchi, and T. Yokoya, Appl. Phys. Lett. 98, 082107 (2011).

${ }^{23}$ K. M. Etmimi, J. P. Goss, P. R. Briddon, and A. M. Gsiea, J. Phys.: Condens. Matter 22, 385502 (2010).

${ }^{24}$ A. Zunger, Appl. Phys. Lett. 83, 57 (2003).

${ }^{25}$ A. Chroneos, R. W. Grimes, and H. Bracht, J. Appl. Phys. 106, 063707 (2009).

${ }^{26}$ A. Chroneos, C. A. Londos, E. N. Sgourou, and P. Pochet, Appl. Phys. Lett. 99, 241901 (2011).

${ }^{27}$ S. Bagchi, C. D. Poweleit, R. T. Beeler, J. Kouvetakis, and J. Menendez, Phys. Rev. B. 84, 193201 (2011).

${ }^{28}$ A. Chroneos, C. Jiang, R. W. Grimes, U. Schwingenschlögl, and H. Bracht, Appl. Phys. Lett. 94, 252104 (2009).

${ }^{29}$ H. Katayama-Yoshida, T. Nishimatsu, T. Yamamoto, and N. Orita, J. Phys.: Condens. Matter 13, 8901 (2001).

${ }^{30}$ D. Segev and S. H. Wei, Phys. Rev. Lett. 91, 126406 (2003).

${ }^{31}$ A. Kumar, J. Pernot, A. Deneuville, and L. Magaud, Phys. Rev. B 78, 235114 (2008). 